\title{
Creativity and information search in a problem-solving context
}

\author{
MARVIN KARLINS, ROBERT E. LEE, III AND HAROLD M. SCHRODER \\ PRINCETON UNIVERSITY ${ }^{1}$
}

This study found consistent individual differences in $S s$ information search patterns (questioning frequency and category sampling) across three problem-solving tasks, a relationship between this behavior and Guilford's productivity measure of creativity (Uses for Things), and an independence of both creativity and information request from intelligence.

One popular approach to measuring creativity via psychological testing is to operationally define creativity as proficiency in generating responses to a word or words, such as "uses for things" (Guilford, 1959; Wallach \& Kogan, 1965). In this framework the degree of creativity is a function of the number of responses or products produced. The higher the productivity the higher the creativity score on the examination.

This productivity notion of creativity makes some sense in a problem-solving context. Keeping in the Guilford "generation of alternatives" conception, one might contend that the more questions an individual is able to ask about a given problem the higher the likelihood of his solving it. In other words, the more active an individual is in his information search of a given problem domain, the greater his chances will be of finding the problem's best possible answer. Before, however, one can determine if more active information search within a given problem leads to a more successful solution of that problem, it must first be determined if there are individual differences in this information seeking propensity across several problem-solving tasks and, further, if this activity correlates with the score on a paper and pencil test of creativity that is based on the number of responses produced. The emphasis of this study, then, is not on the end product of problem-solving-the answer -but rather on the earlier phases of problem-solving -the ways individuals seek information in solving a problem.

To study the question of whether the ability to generate responses on a paper and pencil test of creativity would correlate with the ability to generate questions relevant to a specific problem and, further, whether such information search would be evidenced across different problem situations, three problem-solving tasks were created. They shared two common features: (a) Each task required $S$ to generate questions in solving a problem; (b) Each task was relevant and involving to $S$ (each problem dealt with the Peace Corps, and all Ss were Peace Corps volunteers). The three tasks were:

(1) The Community Development Exercise (CODE) (Karlins, 1967): In this task each $S$ is asked to imagine he is a Peace Corps volunteer assigned to build, in a cooperative effort with the local natives, a hospital on an isolated South Seas island. He is informed that a similar task had been attempted before and failed. Utilizing his information search prerogatives, $S$ is expected to learn about the culture of the islanders he will be living with-asking enough questions about the island and its inhabitants to offer his opinion of the best possible way to get native approval and cooperation in building the hospital.

(2) The Dimension Extract Test (DET) (Lee, 1966): In this problem-solving situation, $S$ is asked to pretend that he must choose a fellow Peace Corps volunteer to help the natives of a local village with their agricultural problems. He has the chance of acquiring from Peace Corps headquarters biographical reports of four possible candidates, but it is his job to tell the national office what information to include in the inventories. His problem is to list all items of information about an applicant that he would consider relevant (information he would ask about each applicant) for making a decision.

(3) Teaching Overseas Programs (TOP) (Schroder, Karlins, \& Lee, 1966): In this final question asking test, $S$ again takes the role of a Peace Corps volunteer and is asked to discover the best possible way to develop English teaching departments in the major secondary schools of his assigned country. $S$ is requested to write down all the questions he would ask in order to gain the relevant information needed to carry out his assignment successfully.

In the CODE and DET, $S$ received two performance scores-the number of questions asked and the number of categories searched (the number of informational domains sampled by $S$ in his information request). The categories were determined by $E$ and it was he who placed the questions into the categories after $S$ had asked them. S received credit for information search in a given category if he asked one or more questions in that information domain. In the CODE the categories were based on the cultural categories developed by the Human Relations Area Files (Murdock et al, 1950); in DET they were formulated from a categorization of all Ss' responses into a limited number of categories. On the TOP problem task, only the number of questions asked was calculated for each S.

The hypotheses of the study were: (1) There will be no significant correlation between Ss performance on a standard intelligence measure and his (a) production of Uses for Things on a creativity test, 2 and (b) 
Table 1. Correlations Between the Experimental Tasks

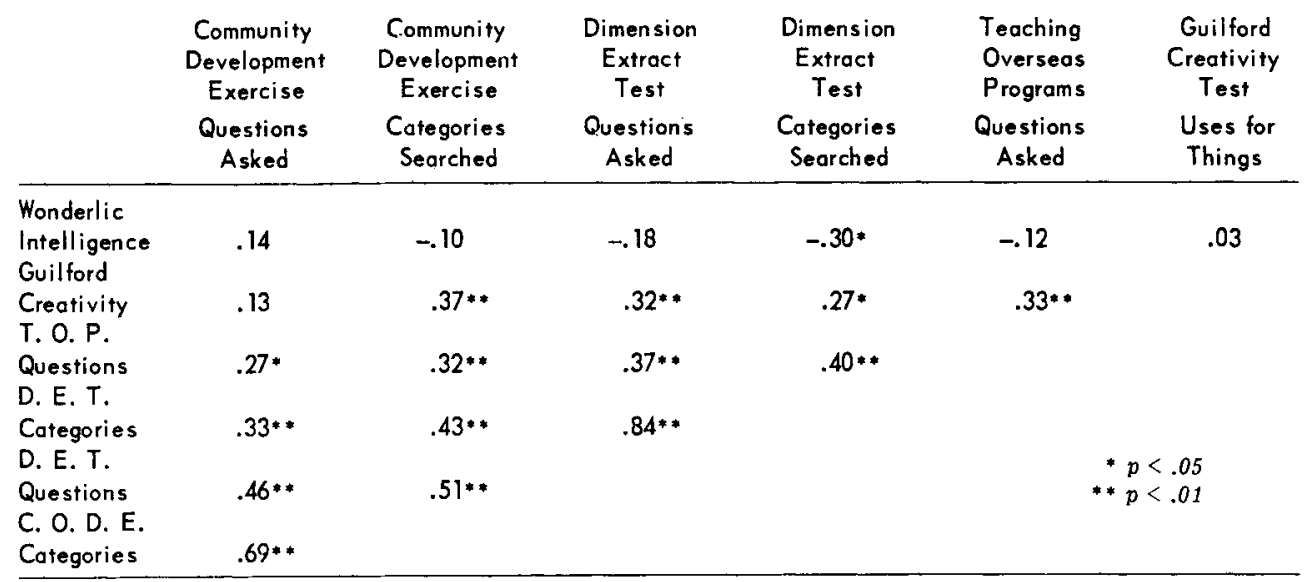

questioning frequency and category search in three different problem-solving tasks. (2) There will be significant correlations between S's performance on the Uses for Things creativity measure and his production of questions and extent of category sampling in three different problem-solving tasks. (3) There will be a cross-task consistency in S's information search behavior on the three problem-solving tasks, reflected in significant correlations between his performances on the CODE, DET, and TOP tasks.

Subjects

65 male and female Peace Corps volunteers, primarily college graduates in their early 20 's, were utilized in the study.

\section{Procedure}

The following tests were administered to Ss over a two-day schedule: (1) Wonderlic Personnel Inventory, an intelligence test that correlates .87 with the Otis Intelligence Scale; (2) Guilford's Uses for Things test of creativity; (3) Community Development Exercise (60 min); (4) Dimension Extract Test (15 min); (5) Teaching Overseas Program Test (15 min).

\section{Results}

Table 1 presents the correlation matrix relevant to the experimental hypotheses. The results were in general support of the hypotheses. The following statements summarize the statistical findings of the study: (1) Intelligence (with one exception in the negative direction) did not correlate with either Guilford's test of creativity or the three problem-solving tasks. In general, there were small negative relationships between Wonderlic scores and performance on CODE, DET, and TOP. (2) In four out of five cases there were significant correlations between S's performance on Guilford's creativity measure and information search in the three problem-solving tasks. (3) In all cases there were significant correlations between S's performance across the three problem-solving tasks. Discussion

The findings of the present investigation support the notions that: (a) Intelligence is not related to a person's ability to generate responses on a test of creativity or his questioning frequency and category sampling on three problem-solving tasks; (b) The ability to produce a number of uses for things on Guilford's test seems to be related to the ability to display an active and diversified information search in selected problem-solving tasks; (c) There seems to be some transsituational consistency in the way individuals search for information in the initial phases of solving a problem, as reflected in Ss' correlated behavior across CODE, DET, and TOP.

It is interesting to note that such "esoteric" activity as being able to name several uses for a brick seems to have its practical side-such enumerative behavior is also displayed when $S$ is seeking a solution to a specific problem. It will be the task of later studies to carry the findings of the investigation further and see if differences in questioning frequency and category sampling lead to more effective problemsolving behavior.

\section{References}

Guilford, J. P. Three faces of intellect. Amer. Psychologist, 1959, 14, 469-479.

Karlins, M. Conceptual complexity and remote associative proficiency as creativity variables in a complex problem-solving task. J. Pers. soc. Psychology, in press.

Lee, R. E. A description of the Dimension Extract Test. Unpublished manuscript, Princeton University Social Psychology Library, 1966.

Murdock, G. P., Ford, C. S., Hudson, A. E., Kennedy, R., Simmons, L. W., \& Whiting, J. W. Outline of cultural materials (3rd ed.). New Haven: Human Relations Area Files, Inc., 1950.

Schroder, H. M., Karlins, M., \& Lee, R. E. A description of the Teaching Overseas Programs Test. Unpublished manuscript, Princeton University Social Psychology Library, 1966.

Wallach, M. A., \& Kogan, N. Modes of thinking in young children: a study of the creativity-intelligence distinction. New York: Holt, 1965.

\section{Notes}

1. This study was supported through the financial assistance of the Peace Corps.

2. This expectation is based on previous findings of other studies which have shown intelligence and creativity to be uncorrelated. 